\title{
CO-37: Estado y planificación inca en Collahuasi (Provincia de Iquique, I Región, Chile)
}

\author{
Alvaro Romero G. ${ }^{1}$ y Luis Briones M. ${ }^{2}$
}

\section{RESUMEN}

Se presenta un asentamiento altiplánico, ubicado sobre los $4.200 \mathrm{~m} . \mathrm{snm}$, contemporáneo a la expansión del Tawantinsuyu en el Collasuyu. Este asentamiento se extiende por aproximadamente $4.500 \mathrm{~m}$, formando un heterogéneo conjunto arquitectónico, que fue analizado mediante técnicas cuantitativas y cualitativas con el objetivo de proponer categorías de planificación del espacio. Los resultados indican que este asentamiento presenta una planificación de características arquitectónicas inca, utilizándose como enclave de múltiples funciones ligadas con la producción minera.

\section{ABSTRACT}

An altiplanic site located at the $4.200 \mathrm{~m} . \mathrm{snm}$, contemporary to the Inca expansion in the Collasuyu is presented. This settlement extends for approximately $4.500 \mathrm{~m}$ forming a heterogeneous architectural compound that was analyzed by means of quantitative and qualitative techniques to propose categories of planning the space. The results indicate that this settlement presents an inca architectural planning. This site is an enclave used for multiple bound functions linked to the mining production.

1 Departamento de Arqueología y Museología, Universidad de Tarapacá, Arica. E-mail: aromero@cabuza.museo.uta.cl

2 Departamento de Arqueología y Museología, Universidad de Tarapacá, Arica. E-mail: lbriones@ cabuza.museo.uta.cl

\section{Introducción}

$\mathrm{El}$ asentamiento arqueológico CO-37 fue reconocido años atrás durante un estudio de impacto ambiental en el área de la actual minera Collahuasi (Núnez $1995 \mathrm{Ms}$ ) y fue dado a conocer brevemente junto a una revisión general del problema inca en los ambientes despoblados del norte de Chile (Lynch y Núñez 1994). De los 10 sitios arqueológicos correspondientes a los períodos Tardío e Intermedio Tardío prospectados en el citado estudio (Figura 1), destaca de sobremanera este asentamiento por una serie de características, entre ellas su gran extensión, buen estado de conservación, monumental envergadura arquitectónica y una ubicación a más de 4.200 m.snm, en pleno ambiente altiplánico.

Este trabajo demuestra a través de un estudio técnico y de uso del espacio la dimensión arquitectónica de estilo incaico que lleva a concluir la existencia de una planificación con características estatales y multifuncionales.

\section{Descripción General de CO-37}

El sitio de CO-37 se ubica en la quebrada de Yabricoyita (UTM: 535.700 E / 7.686.100 N), lugar donde el encajonado curso de dirección oesteeste comienza a abrirse hacia la hoya del salar de Michincha y va formando una suave planicie donde vemos erguirse el imponente cerro Pabellón del Inca $(5.100 \mathrm{~m} . \mathrm{snm})$ divisor y mirador natural de las cuencas de Michincha y Coposa.

La superficie del terreno es bastante rocosa, con piedras volcánicas de variados tamaños y formas, 
que van desde las denominadas piedras lajas hasta bolones de aristas cuadrangulares. La vegetación nativa suele ser sensible a los regímenes de precipitaciones del altiplano, los cuales durante los años de nuestras expediciones conformaron un denso manto de tolas y pajonales.

El área total del asentamiento $\mathrm{CO}-37$ suma 4.900 $\mathrm{m}$, con un largo aproximado de $450 \mathrm{~m}$ siguiendo el recorrido este-oeste de la quebrada Yabricoyita, $\mathrm{y}$ un ancho de casi $380 \mathrm{~m}$ en dirección norte-sur (Figura 2).

Los 55 conjuntos arquitectónicos ${ }^{3}$ que forman el asentamiento están construidos en su totalidad con muros pircados, con un aparejo principalmente rústico, sin mortero o argamasa. No hay evidencias de otros materiales constructivos de uso prehispánico, tales como maderos o paja, pero se observan reutilizaciones recientes de algunas unidades con maderos aserrados y latones.

Las características formales y su arreglo topográfico permiten establecer una división del asentamiento en nueve sectores arquitectónicos (Figura 3). El sector A, corresponde a dos conjuntos de forma poligonal recta de tamaño similar. El sector $\mathrm{B}$, involucra los dos conjuntos de mayor área y de forma más compleja. El sector $\mathrm{C}$, agrupa un único conjunto de 11 unidades circulares muy similares, distribuidas en una particular forma de media luna. El sector D, comprende conjuntos simples de una o dos unidades de forma rectangular o circular que se ubican muy cercanos al cauce seco de la quebradilla. El sector E, consiste en varios conjuntos de diversas formas y tamaños que se ubican algo alejados del núcleo del sitio. Frente al sector E, en la parte norte del cauce seco, se ubi-

3 En este trabajo se utilizan dos unidades de análisis. La primera corresponde a las unidades espaciales. implicando una planificación arquitectónica de espacios delimitados para fines culturales específicos. Esta unidad puede ser equivalente a la unidad "recinto" de la mayoría de las descripciones arquitectónicas. Por otro lado. los coniuntos arquitectónicos. implica un segundo nivel de planificación, que es el agrupamiento de diferentes unidades espaciales de acuerdo a consideraciones culturales. Este agrupamiento se define como un arreglo de una o más unidades espaciales que comparten muros. can una serie de conjuntos arquitectónicos de forma simple y compuestos por una sola unidad espacial, se trata del sector F. El sector G corresponde a un sólo gran conjunto que parece ser un corral post hispano. El sector $\mathrm{H}$. encierra conjuntos muy pequeños y de casi nula evidencia arquitectónica. Finalmente el sector I, involucra dos conjuntos cuadrangulares simples que parecen corresponder a cimientos de arquitectura subactual.

De acuerdo a esta división a priori, los análisis no considerarán los sectores $\mathrm{G}, \mathrm{H}$ e I, por tratarse de sectores que fueron ocupados principalmente después del contacto hispano.

\section{Arquitectura y rasgos de planificación espacial}

El asentamiento CO-37 resultó ser bastante heterogéneo en lo que respecta a tamaño, forma y complejidad o número de unidades espaciales que cada uno de los conjuntos arquitectónicos posee.

El área de cada uno de estos 55 conjuntos arquitectónicos muestra una variación que va de los dos a los $2.500 \mathrm{~m}$, mientras que un $74 \%$ del total del área construida corresponde a los dos conjuntos más grandes. Por otra parte, el número de unidades espaciales que cada conjunto posee varía entre 1 y 34 .

Los conjuntos arquitectónicos con una sola unidad espacial (mono componentes) alcanzan más del $60 \%$ de la muestra, y también suman un área importante en el sitio, ya que presentan la tercera mayoría de área ocupada después de dos unidades con 28 y 35 unidades espaciales.

De acuerdo al número de unidades espaciales de los conjuntos arquitectónicos, se observa que hacia el noroeste del sitio se emplazan la mayoría de los conjuntos arquitectónicos mono componentes. Los conjuntos de dos unidades espaciales se ubican en la parte norte del sitio, mientras que los conjuntos de más de 10 unidades se ubican en la parte SE de CO-37. Por otra parte, los conjuntos de entre 3 y 10 unidades espaciales se disponen de manera regular en el sitio.

\section{Unidades espaciales}

Un análisis de la distribución de la variable área 
en las 161 unidades espaciales estudiadas, nos permite notar que el tamaño presenta un rango entre 0,25 y $1.356 \mathrm{~m}$. Además, un $50 \%$ de las unidades se concentra entre los 2 y $5 \mathrm{~m}$, la media obtenida es de $24 \mathrm{~m}$ y la desviación estándar es de 112 , denotando, con todo esto, un comportamiento bastante heterogéneo del área de las unidades espaciales.

Por otro lado, un segundo análisis que considera sólo las 30 unidades espaciales mono componentes señala una gran homogeneidad, ya que la media alcanza los $6 \mathrm{~m}$ y la desviación estándar sólo es de 12,5. Igualmente, más de un $70 \%$ de estas unidades espaciales tiene un área entre 0,25 y 6 $\mathrm{m}$.

Siguiendo con el análisis, se ha dividido la variable área en ocho rangos, cuya definición y com- portamiento son descritos en la Tabla 1.

Vemos que los dos primeros rangos agrupan más del $80 \%$ de las unidades espaciales. El rango 6 tiene una interesante frecuencia que lo hace aparecer como una medida estándar posiblemente utilizada para alguna finalidad espacial, como se verá más adelante.

La distribución espacial de estos rangos se muestra en la Figura 4. Los sectores A y B se caracterizan por poseer unidades espaciales con una gran variedad de tamaños, mientras que los sectores $C$, $D, E$ y $F$ poseen casi únicamente unidades de rangos 1 y 2 .

Con respecto a la forma de la planta de las unidades espaciales, notamos una distribución diferencial, que se expresa claramente en la Tabla 2.

Tabla 1. Distribución rangos de área

\begin{tabular}{lllr}
\hline & RANGO & N & \% \\
\hline R1 & menos de $4 \mathrm{~m}$ & 68 & $42,2 \%$ \\
R2 & $4-11,9 \mathrm{~m}$ & 69 & $42,9 \%$ \\
R3 & $12-29,9 \mathrm{~m}$ & 8 & $5,0 \%$ \\
R4 & $30-74,9 \mathrm{~m}$ & 6 & $3,7 \%$ \\
R5 & $75-109,9 \mathrm{~m}$ & 2 & $1,2 \%$ \\
R6 & $110-499,9 \mathrm{~m}$ & 7 & $4,3 \%$ \\
R7 & $500-999,9 \mathrm{~m}$ & 0 & $0,0 \%$ \\
R8 & más de 1000 m & 1 & $0,6 \%$ \\
\hline \multirow{2}{*}{ TOTAL } & 161 & $100,0 \%$ \\
\hline
\end{tabular}

Tabla 2. Distribución de la forma de unidades espaciales según sectores

\begin{tabular}{lccccc}
\hline SECTORES & N NN. ESPACIALES $^{\text {CIRCULAR }}$ & IRREGULAR & $\begin{array}{c}\text { CUADRANGULAR } \\
\text { RECTANGULAR }\end{array}$ & $\begin{array}{c}\text { IRREGULAR DE } \\
\text { LADOS RECTOS }\end{array}$ \\
\hline A & 12 & & & $75,0 \%$ & $25,0 \%$ \\
B & 67 & $16,4 \%$ & $13,4 \%$ & $55,2 \%$ & $14,9 \%$ \\
C & 11 & $100,0 \%$ & & $65,0 \%$ & $5,0 \%$ \\
D & 20 & $25,0 \%$ & $5,0 \%$ & $37,5 \%$ & \\
E & 16 & $56,3 \%$ & $6,3 \%$ & $2,9 \%$ & $\mathbf{8 , 7 \%}$ \\
F & 35 & $94,3 \%$ & $2,9 \%$ & $\mathbf{4 1 , 0} \%$ & \\
\hline TOTAL & $\mathbf{1 6 1}$ & $\mathbf{4 2 , 9 \%}$ & $\mathbf{7 , 5 \%}$ & \\
\hline
\end{tabular}


El sector A posee exclusivamente recintos cuadrangulares o poligonales rectos. El sector B, también presenta una mayoría de recintos cuadrangulares, como también algunos recintos de forma circular e irregular. El sector $C$ posee 11 unidades circulares integrando una unidad arquitectónica en forma de medialuna, una forma bastante peculiar, no sólo para el sitio, sino también en los Andes.

El sector D posee similar proporción de unidades circulares y cuadrangulares pero con una leve mayoría de circulares, mientras que en el sector $\mathrm{E}$ ocurre lo contrario. El sector $F$ posee una clara mayoría de unidades de forma circular.

\section{Tipos de muros}

En los seis sectores arquitectónicos en estudio se han descrito 296 muros. La gran mayoría de los muros de CO-37 son de doble hilada (67\%); los paramentos tienen un aparejo, o disposición de elementos, principalmente rústico (76\%); un poco más del $50 \%$ de los muros ofrecen un perfil ataludado, y tan sólo un $26 \%$ se presentan aplomados.

La altura promedio de los muros se ubica entre $\operatorname{los} 40$ y $60 \mathrm{~cm}$, rango en el cual se agrupa sobre el $46 \%$ del total. El ancho promedio de los muros mejor conservados es de casi $50 \mathrm{~cm}$, donde más del $70 \%$ tiene medidas entre 33 y $66 \mathrm{~cm}$.
A continuación se describen estas características según su distribución en los sectores arquitectónicos definidos. En el gráfico de la Figura 5 se observa que el comportamiento del tipo de hilada muestra tres tendencias diferentes. Existiendo un sector que posee exclusivamente muros de hilada doble, el sector $\mathrm{C}$; el tipo de muro doble con relleno y el muro simple poseen una correlación inversa, es decir, a medida que en un sector la presencia de uno de estos tipos es significativa, la presencia del otro disminuye. En los sectores A, B y D prevalece el muro de hilada doble, pero se agrega de manera importante el muro de hilada doble con relleno. En cambio, en los sectores E y $F$ son los muros de hilada simple los que suman una alta presencia.

En la Tabla 3 vemos el comportamiento del tipo de aparejo que, como se dijo, son principalmente de características rústicas.

El paramento de aparejo sedimentario ${ }^{4}$ presenta importantes frecuencias sólo en los sectores A, B y D $(20 \%, 15 \%$ y $5 \%$, respectivamente), mientras que el aparejo indeterminado ${ }^{5}$ posee frecuencias relevantes en los sectores E y F $(24 \%$ y $51 \%$, respectivamente) donde, como se verá más adelante, prevalecen los muros bajos.

Sobre el tipo de aplomo, se debe agregar que el tipo ataludado que se observa frecuentemente en

Tabla 3. Distribución del tipo de aparejo de paramentos según sectores

\section{SECTORES No PARAMENTOS RUSTICO SEDIMENTARIO CELULAR INDETERMINADO}

\begin{tabular}{llrlll}
\hline A & 29 & $79,3 \%$ & $20,7 \%$ & & $4,8 \%$ \\
B & 146 & $79,5 \%$ & $15,8 \%$ & & \\
C & 11 & $100,0 \%$ & & & $24,0 \%$ \\
D & 19 & $94,7 \%$ & $5,3 \%$ & $2,0 \%$ & $51,2 \%$ \\
E & 50 & $74,0 \%$ & & & $13,5 \%$ \\
F & 41 & $48,8 \%$ & & $0,3 \%$ & \\
\hline \multirow{2}{*}{ TOTAL } & 296 & $76,0 \%$ & $10,1 \%$ & & \\
\hline
\end{tabular}

4 El paramento sedimentario corresponde a una forma de disposición más cuidadosa en la construcción y elección de los elementos del muro, donde se busca elevar el muro mediante la colocación de piedras en sucesivos niveles horizontales (ver Castro et al. 1993).
5 Debemos aclarar que el aparejo indeterminado corresponde a muros donde la falta de altura impide discernir el tipo de ordenamiento de los elementos. 
CO-37 es resultado de una mala conservación del muro. La existencia o inexistencia del ataludado nos permite determinar las variaciones de la altura original de los muros. Como dijimos anteriormente, muy pocos muros muestran un ataludado original, tal como los ubicados en los límites del lecho de la quebrada, que parecen reforzar la construcción ante posibles crecidas del río.

Con respecto al comportamiento de la altura de los muros se notan tres grupos de distribución. Se observa en el gráfico de la Figura 6 que los sectores A y B presentan un comportamiento semejante, con similares proporciones (un poco menos del $40 \%$ ) de muros entre los rangos de altura bajo y mediano. Los sectores $\mathrm{C}$ y $\mathrm{F}$, en cambio, poseen importantes proporciones de muros muy bajo y bajo (entre $40 \%$ y $50 \%$ ), con una leve superioridad del muro de altura baja y casi sin ningún muro sobre los $60 \mathrm{~cm}$. Los sectores D y E, al contrario, poseen una proporción substancial de muros bajos (sobre el 60\%) y similares proporciones de muros muy bajos $(10 \%)$ y medianos $(20 \%)$.

El análisis del ancho de la cumbre de los muros nos ofrece otra diferencia entre los sectores, como se desprende de la Tabla 4.

El ancho de rango mediano es el más común en todos los sectores, pero es la segunda tendencia la que varía entre los sectores. Los sectores A, B, C y $D$ poseen además de la alta presencia de muros de ancho mediano, muros con el rango de ancho mayor. En cambio, el sector F, se caracteriza por tener una tendencia hacia muros más angostos. El sector $\mathrm{E}$, posee similares proporciones de muros anchos y angostos (4\% para cada uno).

\section{Tipos de vanos}

Se reconocieron 108 vanos en los sectores arquitectónicos de interés. El cálculo de la densidad de vanos en cada sector se resume en la Tabla 5.

Tabla 4. Distribución de los rangos de ancho de paramentos según sectores

\begin{tabular}{lcccccc}
\hline SECTORES & $\mathbf{N}^{\circ}$ PARAMENTOS & $\begin{array}{c}\text { ANGOSTO } \\
(\mathbf{1 0 - 3 3} \mathbf{~ c m})\end{array}$ & $\begin{array}{c}\text { MEDIANO } \\
\mathbf{( 3 3 - 6 6 )}\end{array}$ & $\begin{array}{c}\text { ANCHO } \\
\mathbf{( 6 6 - 9 9 )}\end{array}$ & $\begin{array}{c}\text { MUY ANCHO } \\
\text { (más de 100) }\end{array}$ & INDETERMINADO \\
\hline $\mathrm{A}$ & 29 & $3,4 \%$ & $62,1 \%$ & $34,5 \%$ & & \\
$\mathrm{~B}$ & 146 & $6,2 \%$ & $77,4 \%$ & $13,7 \%$ & $0,7 \%$ & $2,1 \%$ \\
$\mathrm{C}$ & 11 & & $90,9 \%$ & $9,1 \%$ & & \\
$\mathrm{D}$ & 19 & & $94,7 \%$ & $5,3 \%$ & & $12,0 \%$ \\
$\mathrm{E}$ & 50 & $4,0 \%$ & $80,0 \%$ & $4,0 \%$ & & $7,3 \%$ \\
$\mathrm{~F}$ & 41 & $36,6 \%$ & $53,7 \%$ & $2,4 \%$ & & $4,1 \%$ \\
\hline TOTAL & 296 & $9,1 \%$ & $74,7 \%$ & $11,8 \%$ & $0,3 \%$ & \\
\hline
\end{tabular}

Tabla 5. Distribución de vanos en sectores arquitectónicos

\begin{tabular}{lcccc}
\hline SECTORES & $\mathbf{N}^{\mathbf{0}}$ DE UN. ESPACIALES & $\mathbf{N}^{\mathbf{0}}$ VANOS & \% VANOS & DENSIDAD DE VANOS $^{*}$ \\
\hline A & 12 & 7 & $6,5 \%$ & 0,5 \\
$\mathrm{~B}$ & 67 & 53 & $49,1 \%$ & 0,7 \\
$\mathrm{C}$ & 11 & 10 & $9,3 \%$ & 0,9 \\
$\mathrm{D}$ & 20 & 13 & $12,0 \%$ & 0,6 \\
$\mathrm{E}$ & 16 & 13 & $12,0 \%$ & 0,8 \\
$\mathrm{~F}$ & 35 & 12 & $11,1 \%$ & 0,3 \\
\hline TOTAL & 161 & 108 & $100,0 \%$ & 0,6 \\
\hline
\end{tabular}

- Calculada a partir del número de vanos $(\mathrm{N})$ dividida por el número de unidades espaciales existentes en cada sector arquitectónico. 
Si bien el sector B es el que posee la mayor cantidad de vanos no posee la densidad más alta según el número de unidades espaciales. Lo superan los sectores $\mathrm{C}$ y $\mathrm{E}$, con una incidencia de 0.9 y 0,8 , respectivamente, es decir, en tales sectores casi todas las unidades espaciales poseen un vano.

Otro tipo de variable utilizada fue el tipo de relación espacial que los vanos establecen. Los vanos que relacionan las unidades espaciales con el exterior priman donde existen conjuntos arquitectónicos mono componentes (sector $F$ ), pero ésto también sucede en sectores donde se disponen principalmente conjuntos entre 2 y 9 unidades espaciales (sectores D y E). Otro caso es el que se aprecia en el sector $\mathrm{C}$, donde el conjunto de 11 unidades posee vanos que se relacionan exclusivamente con el exterior.

Enseguida, se exploraron estadísticamente otra serie de variables que dieron escasos resultados debido a la mala conservación de los vanos. En todo caso es interesante observar que estos vanos, en general, no poseen ni dinteles, ni umbrales, y la utilización de grandes piedras monolíticas al modo de jambas es relativamente escasa.

Si bien en el sector F, E y D, observamos previamente una serie de características que nos hablan de una menor complejidad o calidad constructiva, tales como muros de hilada simple, baja altura (menor a $60 \mathrm{~cm}$ ), conjuntos arquitectónicos con menos unidades espaciales y formas de planta principalmente circulares u ovoides, es interesante un aspecto que se nota en la Tabla 6 .

En los sectores ya nombrados, es decir F y E, los vanos construidos con al menos una jamba, son más numerosos que los vanos sin esta característica. Este aspecto se revierte en las unidades de los sectores A, B y especialmente C, donde ningún vano posee jambas.

\section{Comparación entre conjuntos 3 y 5}

Los conjuntos de mayor complejidad arquitectónica son sin duda los del sector B. Estos conjuntos suman 135 unidades espaciales, es decir, casi un $80 \%$ del sitio. A través de cuatro variables se exploró la existencia de diferencias constructivas y de uso del espacio entre tales conjuntos.

En lo que se refiere a la forma de las unidades espaciales, tenemos que en el conjunto 5 existe una amplia mayoría de unidades de forma cuadrangular ( 23 casos, 65\%), en cambio en el conjunto 3 existen tanto unidades cuadrangulares como de forma circular $(39 \%$ y $32 \%$, respectivamente).

Con respecto al rango de área de las unidades, la Tabla 7 nos señala que el conjunto 3 posee una mayor variedad de tamaños, ya que presenta todos los rangos de área.

La diversidad de áreas y forma de las plantas del conjunto 3 nos llevan a establecer un análisis cualitativo. Vemos que éste se estructura a partir de un enorme recinto de rango 8 y una serie de otros recintos de rango 6 . Los recintos de rango 2 y 3 se ordenan aglutinadamente entre sí en zonas marginales. Es evidente que la función de este conjunto 3 se basa en las unidades grandes y no en la relación unidades grandes y pequeñas como parece suceder en el conjunto 5 .

Tabla 6. Distribución de vanos de acuerdo al número de jambas

\begin{tabular}{lcccc}
\hline SECTORES & $\mathbf{N}^{0}$ VANOS & SIN JAMBAS & UNA JAMBA & DOS JAMBAS \\
\hline A & 7 & $85,7 \%$ & $14,3 \%$ & \\
B & 53 & $62,3 \%$ & $22,6 \%$ & $15,1 \%$ \\
C & 10 & $100,0 \%$ & & $15,4 \%$ \\
D & 13 & $53,8 \%$ & $30,8 \%$ & $30,8 \%$ \\
E & 13 & $46,2 \%$ & $23,1 \%$ & $8,3 \%$ \\
F & 12 & $41,7 \%$ & $50,0 \%$ & $14,0 \%$ \\
\hline TOTAL & 108 & $62,0 \%$ & $24,0 \%$ & \\
\hline
\end{tabular}


Tabla 7. Comparación de rangos de área de las unidades espaciales de los conjuntos 3 y 5

\begin{tabular}{|c|c|c|c|c|c|c|c|c|}
\hline \multirow[t]{2}{*}{ RANGOS DE AREA } & \multirow[b]{2}{*}{$\mathbf{N}$} & \multirow[b]{2}{*}{$\%$} & \multicolumn{3}{|c|}{ Cj. Arq.-03 } & \multicolumn{2}{|c|}{ Cj. Arq.-05 } & \multirow[b]{2}{*}{$\%$} \\
\hline & & & AREA & $(\mathrm{m} 2) \%$ & $\mathbf{N}$ & $\%$ & AREA (m2) & \\
\hline RI & 6 & $21,4 \%$ & 13,2 & $0,6 \%$ & 5 & $14,3 \%$ & 13,1 & $1,5 \%$ \\
\hline R2 & 9 & $32,1 \%$ & 60,4 & $2,6 \%$ & 24 & $68,6 \%$ & 178,8 & $20,1 \%$ \\
\hline R3 & 5 & $17,9 \%$ & 80,4 & $3,4 \%$ & 2 & $5,7 \%$ & 30,3 & $3,4 \%$ \\
\hline R4 & 2 & $7,1 \%$ & 85,1 & $3,6 \%$ & 1 & $2,9 \%$ & 63,2 & $7,1 \%$ \\
\hline R5 & 1 & $3,6 \%$ & 96.6 & $4,1 \%$ & 0 & & & \\
\hline R6 & 4 & $14,3 \%$ & 641,2 & $27,5 \%$ & 3 & $8,6 \%$ & 602,2 & $67,8 \%$ \\
\hline R8 & 1 & $3,6 \%$ & 1356,4 & $58,1 \%$ & 0 & & & \\
\hline TOTAL & 28 & $100,0 \%$ & 2333,2 & $100,0 \%$ & 35 & $100,0 \%$ & 887,6 & $100,0 \%$ \\
\hline
\end{tabular}

En el conjunto 5 se destaca el rango 2, con casi un $70 \%$ de los casos. Además, el rango 6 en el conjunto 5 suma un área considerable $(67,8 \%)$, rasgo que le confiere al conjunto una disposición espacial mucho más ordenada que el conjunto 3 , donde no destaca la frecuencia de ningún rango de área.

Al volver a observar la Figura 3 vemos que el conjunto 5 se estructura a través de tres unidades espaciales de rango 6 , alrededor de las cuales se conforman casi 30 unidades espaciales que son principalmente de rango 2 (entre 4 y $12 \mathrm{~m}$ ). Se demuestra entonces una planificación constructiva evidente en el conjunto 5 , que utiliza, significativamente, unidades espaciales con medidas estándar específicas (rango 2 y rango 6).

La comparación del tipo de hilada, aplomo y aparejo de los muros no ofrece diferencias significativas, sino más bien muchas semejanzas.

El ancho y alto de muros, en cambio, presenta algunas tendencias diferentes. Los muros del conjunto 3 tienden a ser más angostos, en promedio, que los del conjunto 5. En el conjunto 3 los muros muestran una mayor diversidad en la altura, en cambio, en el conjunto 5 , los muros tienden a concentrarse entre los 40 y $100 \mathrm{~cm}$ de altura, mostrando escasos ejemplos $(8 \%)$ fuera de esos límites.

Con respecto a los vanos, notamos una mayor densidad de éstos en el conjunto 5, donde este índice alcanza 1,02 mientras que en el conjunto 3 , el índice sólo alcanza 0,53 .

\section{Comentarios finales: Funcionalidad y uso del espacio en $\mathrm{CO}-37$}

Hemos partido del supuesto, perfectamente rebatible, que la segmentación propuesta en sectores es equivalente a una división de espacios diferenciados que se utilizó durante el uso prehispánico del asentamiento.

La evidencia trabajada indicaría que $\mathrm{CO}-37$ fue un asentamiento donde se realizaron múltiples funciones, pero todas ellas orientadas principalmente a la explotación minera de este espacio altiplánico. Es necesario señalar que tal conclusión inferida de nuestra recopilación y análisis de datos coincide ampliamente con una anterior interpretación acerca del mismo sitio (Lynch y Nuñez 1994: 162-163). La única forma de entender la enorme inversión en la construcción de este asentamiento es situarla dentro de la cadena incaica hacia la producción de bienes de prestigio, tan útiles como los bienes de subsistencia en una burocracia imperial de las dimensiones del Tawantinsuyu (Earle 1994). La explotación minera fue esencial en la producción de bienes de prestigio de estilo y referencia imperial, los cuales fueron usados como elemento de disuasión política en la incorporación y administración de regiones y grupos humanos.

Este sector altiplánico carece de registros previos de densidad poblacional importante, lo que junto a la magnitud del asentamiento nos lleva a pensar que la institución de los mitimaes o especialistas trasladados por el Estado (Murra 1978 : 230) fue vital para sustentar las diversas tareas que 
involucró el sitio. Los especialistas son un componente esencial para una sociedad con una complejidad social del tipo estatal, bien para la extracción, producción y tráfico del mineral, como para la distribución y tráfico de bienes de subsistencia traídos desde otros lugares del Estado. La diferenciación en cuanto a prestigio y funcionalidad de las distintas especialidades debería ser evidenciado en los diversos sectores propuestos a través de sus características arquitectónicas y arqueológicas.

Los datos arquitectónicos nos indican que el sector A corresponde a un lugar que nos remite de manera clara al Tawantinsuyu. Este referente se expresa principalmente en la planificación en RPC ${ }^{6}$ los muros dobles y dobles con relleno y el aparejo sedimentario de los dos conjuntos arquitectónicos cuadrangulares. Además, estos conjuntos se oponen en su ubicación y su extensión acotada al resto del asentamiento que se extiende hacia el oeste, evidenciando una funcionalidad distinta. Proponemos a modo de hipótesis que este sector se constituyó como núcleo administrativo del asentamiento y no descartamos el uso habitacional de algunos de sus recintos menores por personas de cierta jerarquía. Debemos repetir que no tenemos otra evidencia arqueológica, a parte de la arquitectura, para avalar esta hipótesis.

El sector B comprende dos extensos conjuntos con claras diferencias en cuanto a la planificación de su arquitectura. No se tienen antecedentes para sustentar que ésto se deba a diferencias sincrónicas en la ocupación debido a distintas poblaciones o si se podría explicar como diferentes amplificaciones de la ocupación inca en Collahuasi.

Podemos señalar que el conjunto 5 muestra un patrón arquitectónico más uniforme y apegado a una planificación de líneas incaicas, como el RPC, manteniendo dos medidas de área estándar (rangos 2 y 6) que sirven para ordenar el conjunto. Existen varios estudios que indican que los incas manejaron una serie de medidas para organizar su

6 Raffino señala que este rasgo de planificación es un indicador de primer orden del Tawantinsuyu, entre otros datos simbólicos y funcionales destaca uno estadístico para corroborar esta afirmación: el $69 \%$ de las instalaciones analizadas por su estudio presentan esta característica (1981: 83). imperio, entre las que se incluyen medidas de longitud utilizadas para la planificación arquitectónica (Hyslop 1990:27 y 312 nota 14).

El conjunto 3 muestra un aglutinamiento menos planificado, destacando los recintos grandes, incluso más grandes que los del conjunto 5, que evidencian un uso diferente. Planteamos que el denso uso subactual de este conjunto no es mera coincidencia y se debe a que estos últimos ocupantes aprovecharon una planificación arquitectónica prehispánica orientada a la crianza de animales.

El sector C correspondería a un sistema de silos que aún estaba en construcción, como lo evidencia su doble hilera con una altura no mayor a los $40 \mathrm{~cm}$ y la ausencia de derrumbes y otros restos culturales.

Se plantea que el sector $D$ es un sector residencial de personal especializado en labores administrativas o de tareas específicas en la producción minera. La clara separación de estas unidades de los conjuntos aglutinados, la uniforme calidad constructiva, la complejidad interna o subdivisiones arquitectónicas indicaría la presencia de una población diferenciada, quizás mitimaes provenientes de los reinos altiplánicos incorporados al Tawantinsuyu.

El sector E se nos presenta como una continuación del sector $\mathrm{D}$, con unidades espaciales de similar patrón constructivo. En la superficie del conjunto 9 se encontró una concentración de escoria de fundición y mineral de cobre, junto con probables instrumentos líticos ligados a la molienda del mineral. No tenemos certeza que esta evidencia superficial sea prehispana, pero al menos sabemos que la arquitectura lo es, aunque presenta fuertes indicios de reocupación subactual. Esta escasa evidencia nos hace suponer que los habitantes de las unidades residenciales del sector $E$ fueron especialistas ligados a la fundición de mineral.

Finalmente, el sector F despliega una interesante uniformidad en forma y área de las unidades, donde destaca una que es constructivamente diferente, que al igual que las otras presenta escasa altura pero que es de hilada doble con relleno. Como hemos señalado, este sector no presenta rasgos 
culturales superficiales, como los otros sectores del asentamiento. Su ubicación en la ladera opuesta a una altura mayor que el resto del asentamiento y su estandarizado tamaño y forma nos hacen postular una arriesgada conjetura difícil de contrastar en el futuro: Se trataría de una serie de hornos de fundición que estaban en construcción, similares en forma a los de Quillay, sitio inca del Noroeste argentino (Raffino et al. 1996).

Los hornos detectados en Quillay fueron construidos de barro, de una altura original de $2 \mathrm{~m}$ y de un ancho promedio de la base cercano a un metro. La evidencia en superficie del sitio trasandino es abundante escoria de fundición y carbón, más algunos crisoles o moldes de fundición. Su ubicación en ladera sobre terrazas en lo alto de profundas cárcavas que generan violentos vientos, junto con la abundante leña que rodea los hornos, ayudan a contextualizar el hallazgo argentino (Raffino et al. 1996: 61-63).

En CO-37 pocas cosas se repiten, incluso el diámetro promedio de las unidades sobrepasa el 1,5 m. Aún así pensamos que las unidades del sector F estaban en proceso de edificación por la escasa altura y el nulo ataludado de los muros, y princi- palmente porque otros espacios de CO-37 también estaban en evidente proceso constructivo, como el sector $\mathrm{C}$.

También proponemos que los grandes corrales del sector B, más que servir a la crianza de camélidos, que pueden transitar libremente por el bofedal, sirvieron para concentrar el combustible que se necesitaba utilizar para la fundición de metales, en este caso, guano de camélido. Pensamos que este sistema de futuras huayras iba a reemplazar un patrón más disperso de explotación y fundición que estaba funcionando antes del Tawantinsuyu, pero que aún no dimensionámos completamente en nuestras prospecciones del área ( Núñez en este volumen).

Las excavaciones que se están realizando en el sitio nos ofrecerán nuevos antecedentes para confirmar o rebatir muchas de las hipótesis propuestas acá, relativas a funcionalidad y origen de las poblaciones que hicieron uso del asentamiento. Aunque también importantes sugerencias expuestas en esta ocasión, relativas a aspectos de planificación que no fueron concluidas por los constructores originales, difícilmente podrán ser contrastadas mediante la metodología arqueológica.

\section{BIBLIOGRAFIA}

CASTRO, V.; F. MALDONADO y M. VASQUEZ. La arqui1993 tectura del "Pukara" de Turi. Actas del XII Congreso Nacional de Arqueología Chilena. Boletín del Museo Regional de la Araucanía, Tomo II, $\mathrm{N}^{\circ}$ 4:79106. Temuco.

EARLE, T. Wealth finance in the Inka empire: Evi1994 dence from the Calchaqui Valley, Argentina. American Antiquity 59(3): 443460.

HYSLOP, J. Inka settlement planning. Austin:Uni1990 versity of Texas Press, Austin.

LYNCH, T y L. NUÑEZ. Nuevas evidencias inkas entre Kolla1994 huasi y Río Frío. Estudios Atacameños 11: 145-164, San Pedro de Atacama.
MURRA, J. La organización económica del Esta1978[1956] do Inca. Siglo XXI Editores, México.

NUÑEZ, L. Reconocimiento arqueológico área mi$1995 \mathrm{Ms}$ na. Estudio de Impacto Ambiental Compañía Minera Doña Inés de Collahuasi. Geotécnica Consultores Ltda.

RAFFINO, R. Los Incas del Kollasuyu. Editorial Ra1981 mos Americana, Buenos Aires.

RAFFINO, R., R. ITURRIZA, A. IACONA, A. CAPPARELLI, 1996 D. GOBBO, V. MONTES y R. VASQUEZ. Quillay: Centro metalúrgico Inka en el noroeste argentino. Tawantinsuyu 2: 59-69. 


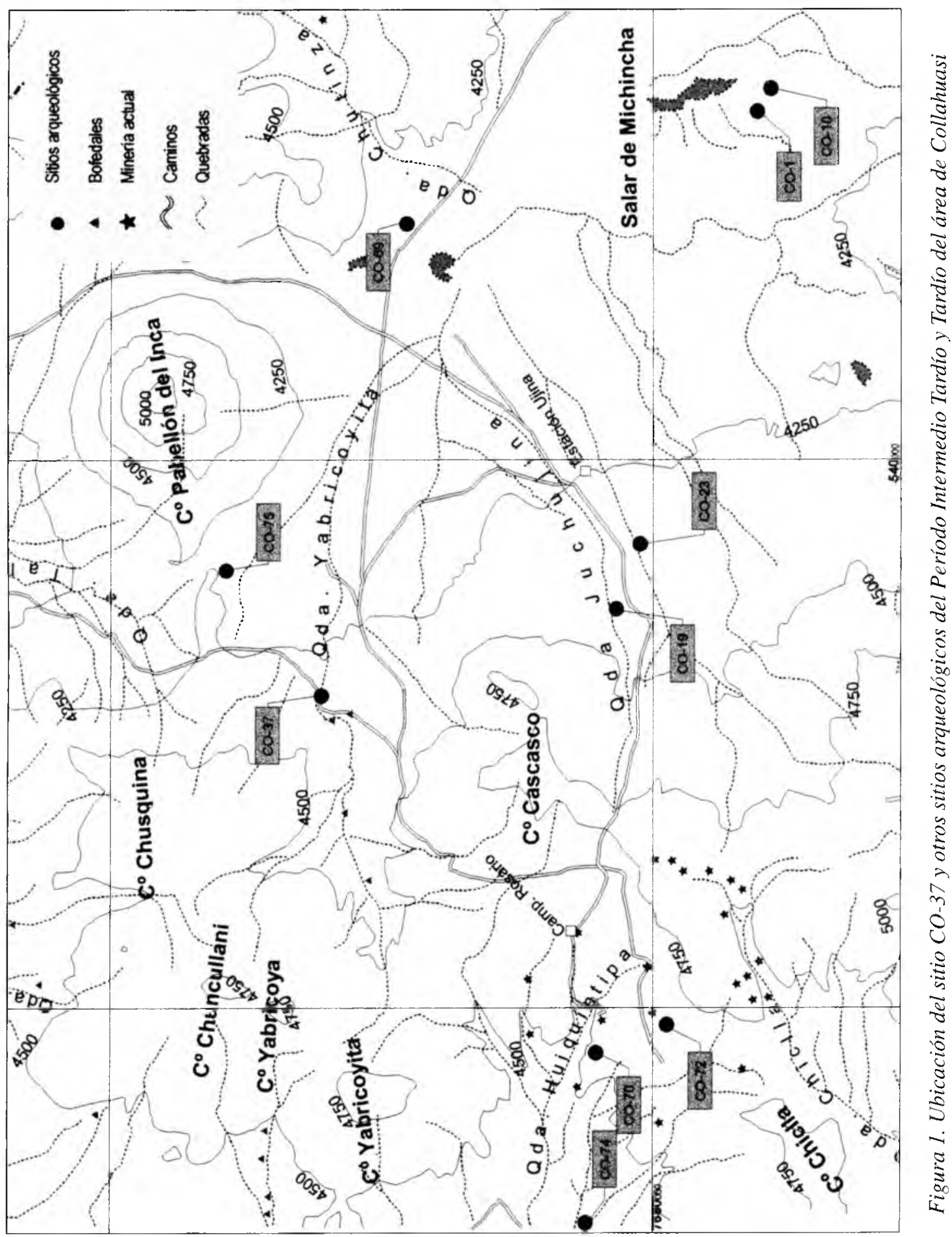




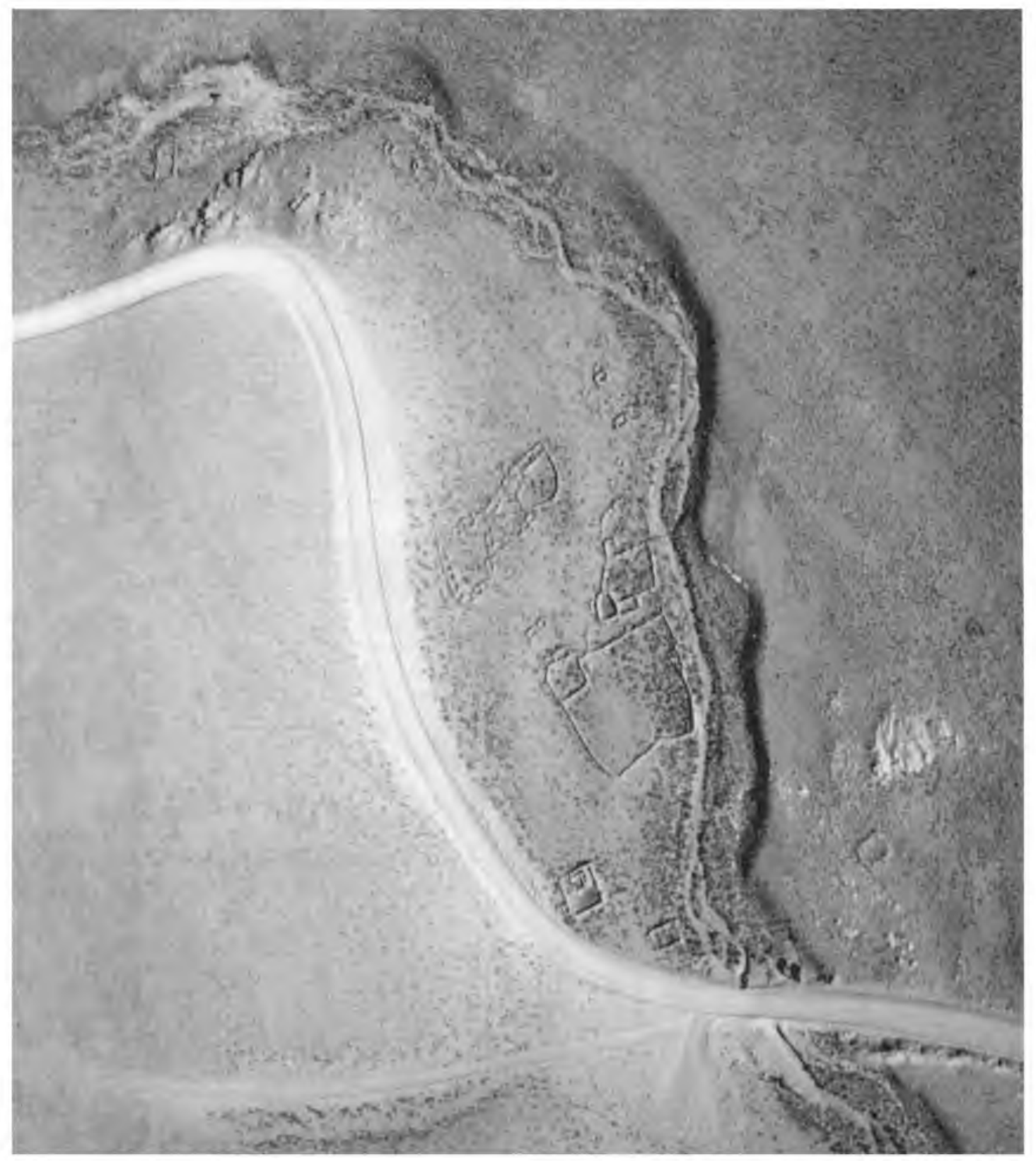

Figura 2. Fotografía aérea de CO-37 


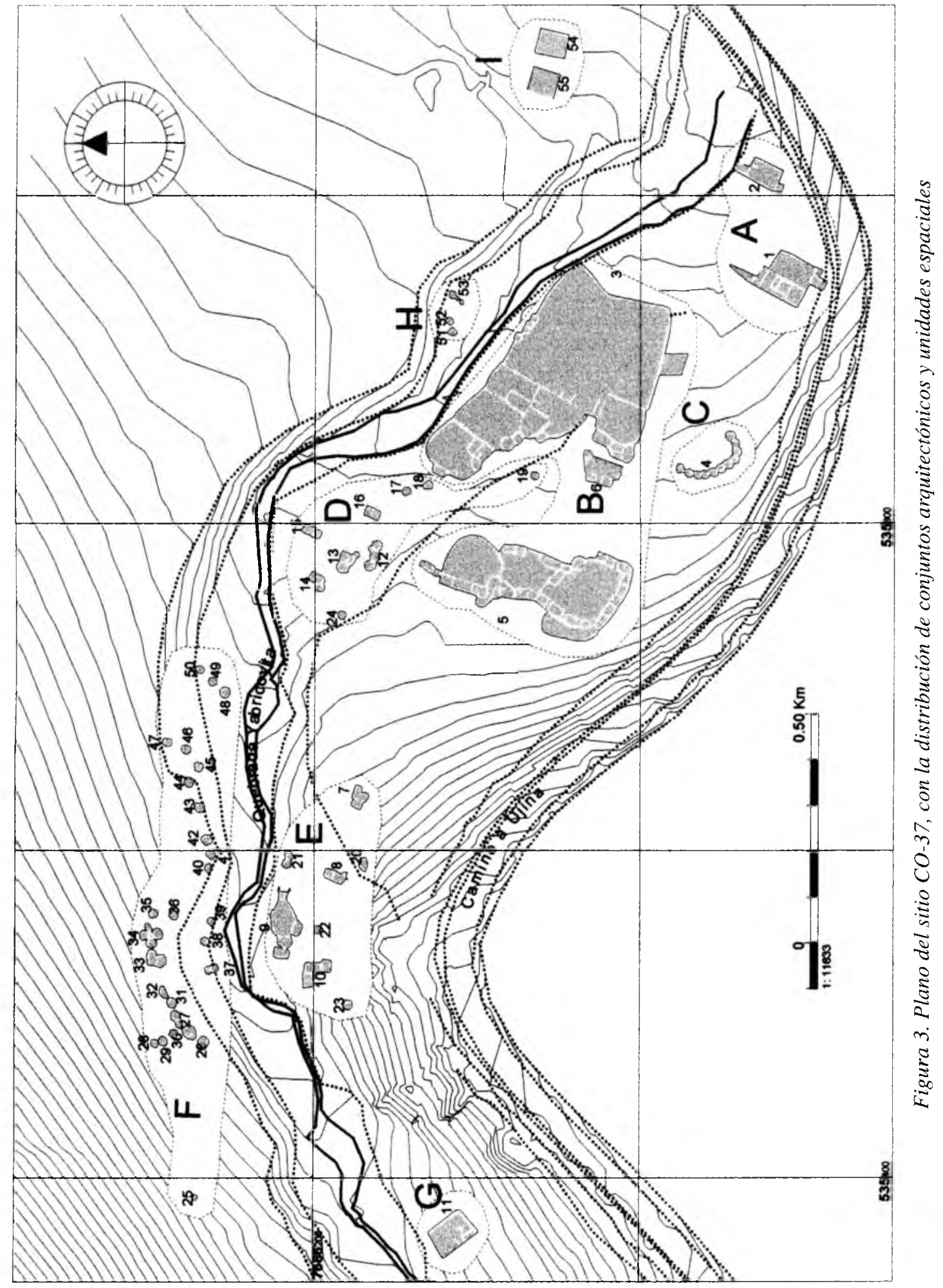




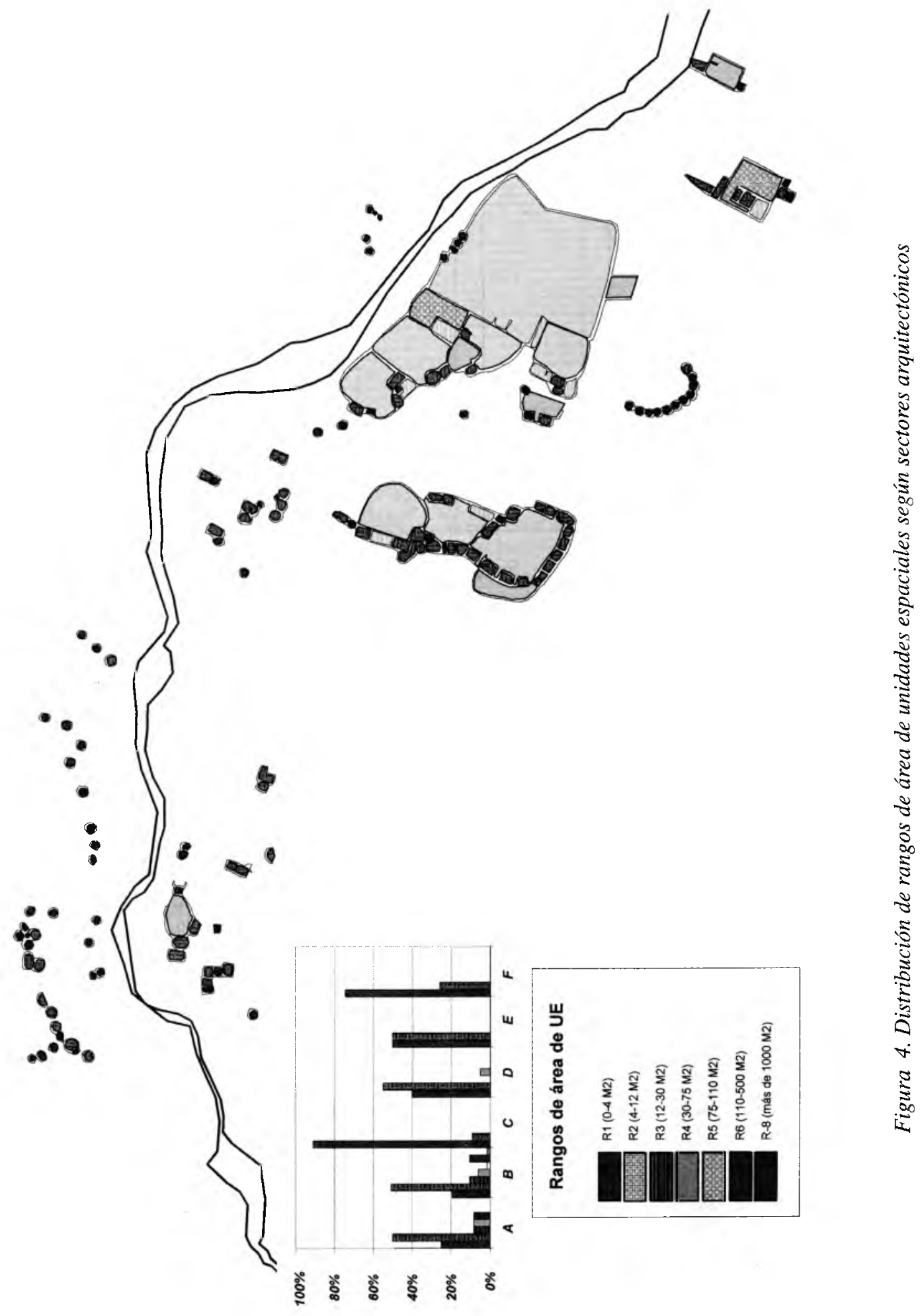




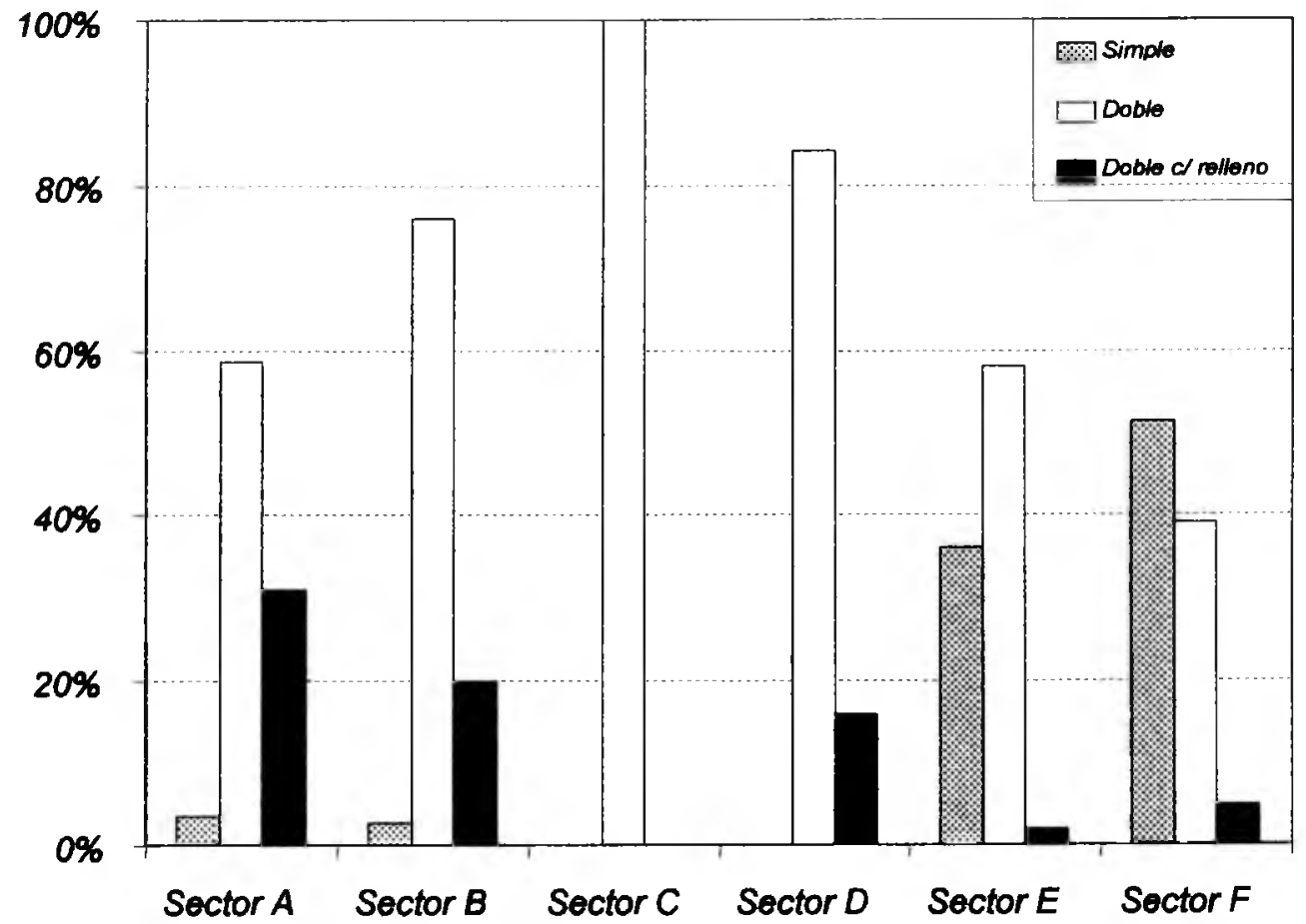

Figura 5. Tipo de hilada de los muros y su distribución en los sectores arquitectónicos

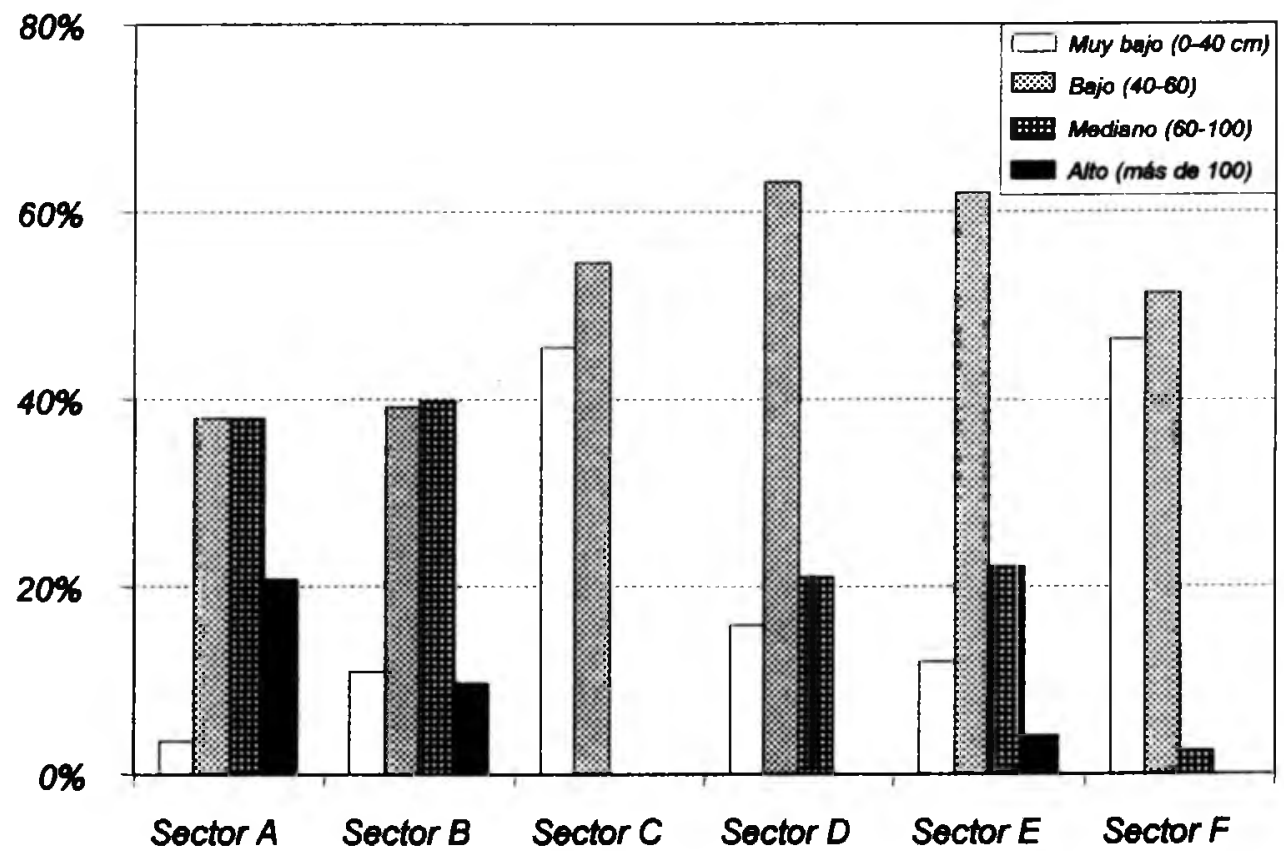

Figura 6. Rangos de altura de muros y su distribución en los sectores arquitectónicos 